DEPÓSITO LEGAL ZU2020000153

Esta publicación científica en formato digital

es continuidad de la revista impresa

ISSN 0041-8811

E-ISSN 2665-0428

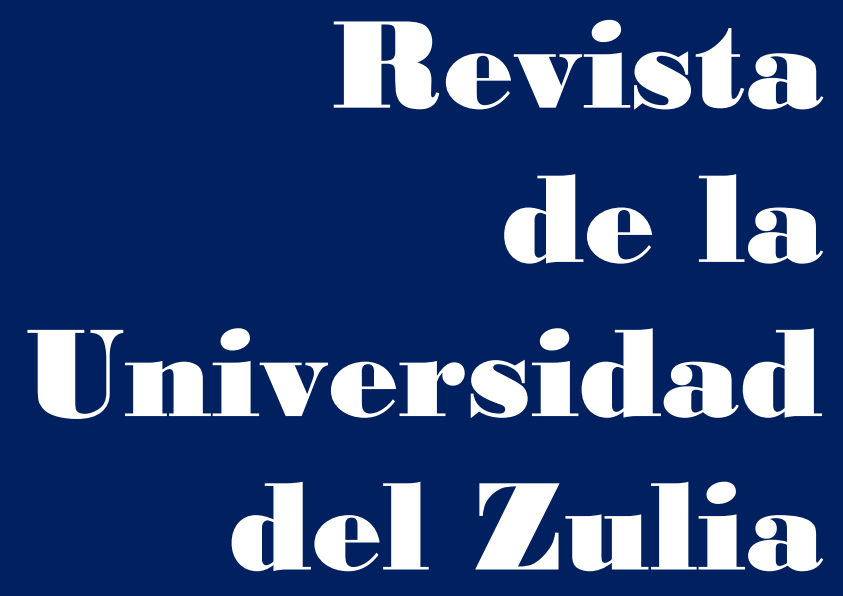

Fundada en 1947

por el Dr. Jesús Emrique Lossada

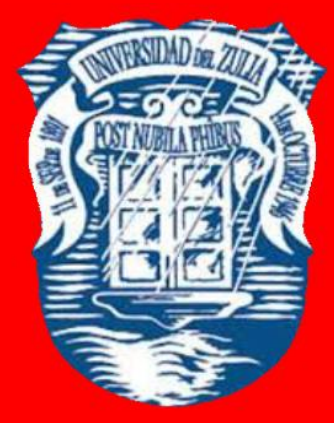

Ciencias

Sociales

y Arte

Año $12 \quad N^{\circ} 34$

Septiembre - Diciembre 2021

Tercera Época

Maracaibo-Veneruela 


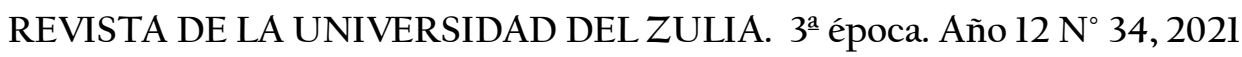

Mikel Ugando Peñate et al.// Modelización financiera aplicada en empresas... 8-28

DOI: http://dx.doi.org/10.46925//rdluz.34.02

\title{
Modelización financiera aplicada en empresas del sector manufacturero en la ciudad de Santo Domingo de los Tsáchilas, Ecuador
}

\author{
Mikel Ugando Peñate* \\ Antonio Villalón Peñate ** \\ Ángel Ramon Sabando García *** \\ Doris Maria Celi Pinza $* * * *$ \\ Félix Sixto Pilay Toala ***** \\ Alba del Pilar Racines Cabrera $* * * * * *$
}

RESUMEN

La presente investigación tiene como objetivo dar a conocer las diversas herramientas de planeación y modelación financiera aplicada en el contexto de las pymes para la determinación de necesidades de financiamiento externo, niveles de activos y realizar predicciones financieras mediante el empleo de los estados financieros, volúmenes de ventas, y razones financieras. La investigación desarrollada en la empresa Reciplacom S.A durante el periodo 2016-2019 tuvo como criterio establecer una política estructurada para la realización de una planeación eficiente y la optimización de recursos mediante el empleo de un enfoque mixto. Para el análisis matemático, estadístico y gráfico se utilizaron las aplicaciones informáticas Microsoft ${ }^{\circledR}$ Excel (2019), y SPSS versión 2l. Se realizó la aplicación de la regresión lineal múltiple utilizando las herramientas estadísticas de Kolmogoro-Smirnov y la autocorrelación de residuos de Durbin Watson lo que permitió determinar los porcentajes de proyección de estados financieros, niveles de activos y excesos de financiamiento externo. Las predicciones se ajustan mejor al modelo ARIMA $(0,0,0)(0,0,0)$. Para concluir, se puede evidenciar que las técnicas de planeación y modelación financiera aplicada ayudan a determinar la salud financiera de la empresa a largo plazo, brindando parámetros eficientes en cuanto a la determinación de los fondos externos requeridos.

PALABRAS CLAVE: Planificación; financiación; política financiera; inversión; datos estadísticos.

*Profesor de la Pontificia Universidad Católica del Ecuador, Sede Santo Domingo, Ecuador, PUCESD. Director del Proyecto de Investigación Grupo Finnovaplan, PUCESD. ORCID: https:/orcid.org/0000-0002-3021-0717. E-mail: upm@pucesd.edu.ec, ugando75@gmail.com

** Miembro del Proyecto de Investigación Grupo Finnovaplan, Pontificia Universidad Católica del Ecuador, Sede Santo Domingo, Ecuador. ORCID: https://orcid.org/0000-0002-5746-1145. E-mail: avillalonp@pucesd.edu.ec, antoniovillalon1987@gmail.com

*** Profesor de la Pontificia Universidad Católica del Ecuador, Sede Santo Domingo, Ecuador, PUCESD. Miembro del Proyecto de Investigación Grupo Finnovaplan, PUCESD. ORCID: https://orcid.org/0000-0003-2696-015X. E-mail: sgar@pucesd.edu.ec

****Profesora de la Pontificia Universidad Católica del Ecuador, Sede Santo Domingo, Ecuador, PUCESD. ORCID: https://orcid.org/0000-0002-1593-5509. E-mail: cpdm@pucesd.edu.ec

***** Profesor de la Pontificia Universidad Católica del Ecuador, Sede Santo Domingo, Ecuador, PUCESD. Miembro del Proyecto de Investigación Grupo Finnovaplan, PUCESD. ORCID: https://orcid.org/0000-0001-7376-3197. E-mail: ptfs@pucesd.edu.ec

****** Profesora de la Pontificia Universidad Católica del Ecuador, Sede Ibarra, Ecuador, PUCESI. ORCID: https://orcid.org/0000-0002-9836-8836. E-mail: adracines@pucesi.edu.ec, albadelpilar@hotmail.com 


\section{Financial modeling applied in companies in the manufacturing sector in the city of Santo Domingo de los Tsáchilas, Ecuador}

ABSTRACT

The present research aims to present the various financial planning and modeling tools applied in the context of SMEs to determine external financing needs, asset levels and make financial predictions through the use of financial statements, volumes of sales, and financial reasons. The research carried out in the company Reciplacom S.A during the period 20162019 had as a criterion to establish a structured policy for the realization of efficient planning and the optimization of resources through the use of a mixed approach. For the mathematical, statistical and graphic analysis, the computer applications Microsoft ${ }^{\circledR}$ Excel (2019), and SPSS version 21 were used. Multiple linear regression was applied using the Kolmogoro-Smirnov statistical tools and the Durbin Watson residuals autocorrelation, which allowed determining the projection percentages of financial statements, asset levels and excesses of external financing. The predictions better fit the ARIMA model $(0,0,0)$ $(0,0,0)$. To conclude, it can be seen that the applied financial planning and modeling techniques help to determine the financial health of the company in the long term, providing efficient parameters in terms of determining the external funds required.

PALABRAS CLAVE: Planning; financing; financial policy; investment; statistical data.

\section{Introducción}

Conforme a las nuevas condiciones económicas y financieras en Ecuador, es de vital importancia que las pequeñas y medianas empresas pongan en práctica estudios de investigación para poder mejorar su situación financiera. De aquí parte la importancia de las decisiones a corto y mediano plazo para el éxito de la empresa y toma de decisiones gerenciales que influyan eficientemente en la obtención de mejores fuentes de financiamiento. Siguiendo este modelo, se manifiestan los relatos teóricos sobre la planeación y modelación financiera en el contexto internacional, técnicas de planeación financiera a corto plazo, determinación de fondos externos requeridos y predicción de niveles de activos mediante la regresión lineal múltiple.

Dentro de esta investigación se considera el financiamiento externo de la empresa para poder tomar decisiones de inversión en función de las operaciones de negocio de la organización, a realizarse tanto en mediano y largo plazo en el país; determinando así los fondos requeridos en el proceso de análisis financiero de acuerdo a la modelación financiera 


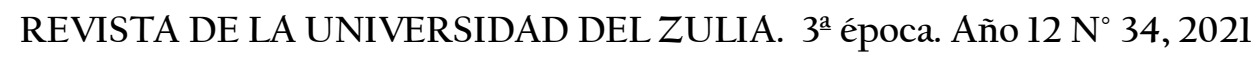

Mikel Ugando Peñate et al.// Modelización financiera aplicada en empresas... 8-28

DOI: http://dx.doi.org/10.46925//rdluz.34.02

aplicada, que es de importancia en la administración moderna y que influirá en el transcurso continuo administrativo de la organización.

Todo lo anteriormente descrito está fundamentado en Córdoba (2012), Domínguez (2011), Fernández (2010) y Gómez (2003), lo que servirá para que la empresa siga aumentando su éxito empresarial, particularmente de acuerdo a las necesidades de financiamiento externo, las cuales están direccionadas bajo la aplicación de la modelación financiera aplicada que contribuye al cumplimiento eficiente de los objetivos generales, para que se administren de mejor manera los recursos financieros de la empresa.

En la ciudad de Santo Domingo de los Tsáchilas las pequeñas y medianas empresas se amplían conforme al desarrollo de la provincia, que cada día crece teniendo más colaboración económica en el contexto nacional e internacional. Para que esto siga manteniéndose, las empresas deben aplicar técnicas y herramientas que permitan prever el futuro de la organización. Se ha tomado como objeto de estudio a la Empresa Reciplacom S.A, identificando como problemática de investigación que, en la misma, no se han implementado a partir de la modelación financiera aplicada, herramientas para la determinación de niveles de activos y requerimientos financieros que incidan eficientemente en el proceso de toma de decisiones de la empresa.

Esta investigación se justifica al poseer una estrecha relación con el eje 2, objetivo 4 del Plan Nacional de Desarrollo 2017-2021 Toda una vida de Ecuador: Consolidar la sostenibilidad del sistema económico social y solidario, y afianzar la dolarización. Garantizar el funcionamiento adecuando de la sostenibilidad macroeconómica y al desarrollo del país. Además, se encuentra direccionado con la línea de investigación de la Pontificia Universidad Católica del Ecuador, Sede Santo Domingo ligada a la administración eficiente y eficaz de las organizaciones para la competitividad local y global, que tributa directamente al Proyecto de Investigación Finnovaplan de la Puce SD y al Plan de desarrollo de Ordenamiento Territorial Santo Domingo 2015 - 2030.

El objetivo general de la investigación se identifica como: La determinación de las necesidades de financiamiento externo, niveles de activos y predicciones financieras para la Empresa Reciplacom S.A., mediante la aplicación de técnicas y herramientas de planeación y modelación financiera aplicada. 


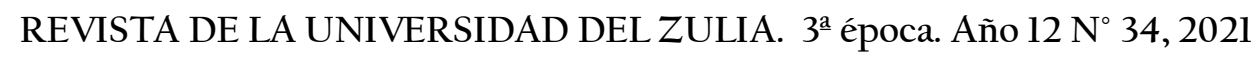

Mikel Ugando Peñate et al.// Modelización financiera aplicada en empresas... 8-28

DOI: http://dx.doi.org/10.46925//rdluz.34.02

\subsection{Planeación y modelación financiera aplicada en el contexto internacional}

Hace varios años, la modelación financiera no era muy tomada en cuenta dentro de países, a excepción de las potencias mundiales; como lo menciona Suárez (2014: 1-2) "Estados Unidos es el líder indiscutible en la planeación financiera y su modelación”. Aportando a esta cita, se considera a este país un modelo que gracias a sus principios ha mantenido un sistema a seguir que se debe ir actualizando de acuerdo con las necesidades que diariamente surgen de las empresas, y poder mejorar su rentabilidad y su estabilidad económica.

Para entender el financiamiento en el contexto mundial se debe tener en cuenta las causas que generan la necesidad de financiamiento, las mismas que surgen en las economías europeas ya que por las guerras, el logro de la estabilidad financiera de ese continente exigía estimular el desarrollo económico y social. Los estados financieros ofrecen información de hechos pasados, son un punto de partida para el desarrollo de un plan financiero a futuro, mediante la utilización de las finanzas y las estrategias de financiamiento.

La modelación financiera crea una guía de lo que se hará en el futuro, es un medio de visualización sistemática del financiamiento que se requiere por parte de las empresas para, en el caso que sea necesario, la adquisición de un activo fijo o la implementación de un nuevo producto hacia el mercado (Ugando et al., 2019: 65-78). Es por ello que se debe elaborar una planeación, para en base a este patrón determinar la viabilidad que tiene la empresa para su posterior evaluación y realización del mismo (Castro, 2017: 17).

Utilizando la información de los estados financieros de años pasados se puede empezar con el análisis, el cual revela los movimientos que se realizan en cuentas de activo y la relación que tengan con los pasivos y el patrimonio o viceversa. Ross et al., (2012) y Van Horne (2014), mencionan como punto de partida el análisis vertical de los estados financieros de un mismo periodo contable; aquí se trata de encontrar la magnitud de las cifras juntamente con las relaciones que tienen las subcuentas con el total de la cuenta principal; las cantidades obtenidas se interpretan en porcentajes permitiendo conocer la estructura de la empresa. El análisis horizontal se hace para ambos estados pertenecientes a varios períodos; se deben tener en cuenta las ponderaciones de los periodos, siendo estas importantes en razón a que generan la condición de los cambios. En las comparaciones históricas es donde se muestran los cambios que han sufrido las diversas cuentas de activos, pasivos y patrimonio que están 


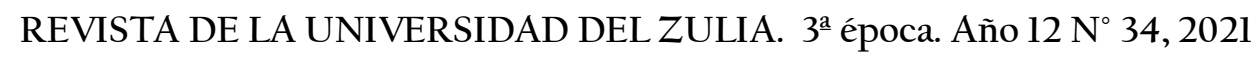

Mikel Ugando Peñate et al.// Modelización financiera aplicada en empresas... 8-28

DOI: http://dx.doi.org/10.46925//rdluz.34.02

definidos en porcentajes (Van Horne, 2010). Estos criterios concuerdan con los resultados de investigación obtenidos por Ugando et al. (2021).

\subsection{Técnicas de planeación financiera a corto plazo}

Mediante las proyecciones de ingresos y desembolsos futuros, la empresa puede obtener el presupuesto de caja de varios periodos. Ross et al. (2012) exponen un modelo general para poder realizar el pronóstico de efectivo: empezando por el pronóstico de ventas, el cual es fundamental para las proyecciones de efectivo de la empresa; este se puede basar en un análisis interno, externo o ambos.

Siguiendo con el modelo, el pronóstico de desembolso de efectivo se efectúa después de realizar las proyecciones de ventas; este desembolso procede de todas las reparticiones de efectivo que ocurren dentro de un periodo contable de la empresa, que se fluctúan generalmente por las ventas de los productos, reflejado también en el costo de ventas, salarios y las rotaciones de inventarios, todo este grupo de rubros son los que generan el mayor movimiento de dinero por el relativo intercambio entre producto y efectivo. A esto se puede adicionar los gastos de ventas por el cumplimiento de las metas que se establecen por un determinado monto de productos vendidos.

De la misma manera hay egresos que no se toman en cuenta, como lo son: las compras en efectivo, cuentas por pagar que han sido canceladas, dividendos, los sueldos a trabajadores y salarios a empleados, intereses sobre los pasivos, el pago de préstamos y de bonos, etc. La necesidad de financiamiento ocurre cuando el saldo de la caja final es menor que el saldo mínimo de efectivo, de aquí se parte con la toma de decisiones de la empresa para poder cubrir el déficit existente. Al contrario, si existe un excedente cuando el saldo final es mayor al mínimo se puede usar este exceso de dinero en inversiones a corto plazo; si bien al existir cualquiera de estas dos opciones (superávit o déficit) deberán ser debidamente ajustados para obtener el saldo final de caja.

Tomando como referencia lo que mencionan Van Horne (2014) y Ross et al., (2014) en sus obras, con el Estado de Resultado Pro Forma se realizan proyecciones de los resultados que obtendrán en el futuro; dentro del estado juega un papel sumamente importante el pronóstico de las ventas, ya que de aquí se pueden proyectar los ingresos y costos de las actividades económico-financieras en los periodos contables. 


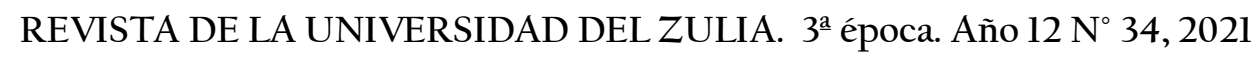

Mikel Ugando Peñate et al.// Modelización financiera aplicada en empresas... 8-28

DOI: http://dx.doi.org/10.46925//rdluz.34.02

Este pronóstico es un resumen de los ingresos y egresos esperados en el futuro, determinando el ingreso o pérdida neta. Como paso siguiente se proyectan los gastos de ventas, administrativos y generales; estos gastos tienen estimaciones bastante precisas por el motivo de que se generan con anticipación. Una vez determinado todos los rubros del estado se pueden precisar los requerimientos financieros externos necesarios mediante el método denominado balance general proyectado.

El Balance General Pro Forma es una copia íntegra del valor contable de la empresa dentro de un período específico; el balance general está estructurado por activos, pasivos y capital, distribuidos de manera horizontal como vertical, mostrando cómo la empresa se financia y lo que la empresa posee. Una vez que se obtienen los estados financieros proyectados, el encargado del departamento de finanzas puede analizar y determinar los ajustes necesarios; por ejemplo, si las utilidades que reflejan son muy bajas se puede proceder a ajustar los costos para reducirlos y aumentar el porcentaje de las utilidades; en el caso de las cuentas por cobrar si estas son muy altas se puede recurrir a crear un nuevo sistema de créditos y cobranzas donde se propongan diferentes políticas para obtener la recuperación más rápida del dinero.

\subsubsection{Fondos Externos Requeridos (FER) y crecimiento}

Ross et al. (2012: 68) mencionan que "El financiamiento externo necesario y el crecimiento están relacionados entre sí". Si no intervienen otros factores, mientras más alta sea la tasa de crecimiento de ventas o activos, mayor va a ser la necesidad de financiamiento externo. Lo primero que se debe hacer es establecer la relación entre el FER y el crecimiento. Con esto se mantiene que, si las ventas son proyectadas, se toma en cuenta que las deudas también aumentan, pero en el pasivo no existe una utilidad superior, determinando así el fondo externo requerido entre un período y otro.

Es obvio que la variable financiamiento externo y la variable crecimiento van relacionados entre sí, así como se expresó anteriormente; se entiende que cuanto más elevada sea la tasa de crecimiento en ventas mayor será la necesidad de financiamiento, ya que para llegar a un crecimiento financiero se debe establecer en primer lugar los estados de resultados y el balance general proyectado, obteniendo así una idea más clara de la necesid ad que tienen las empresas. 


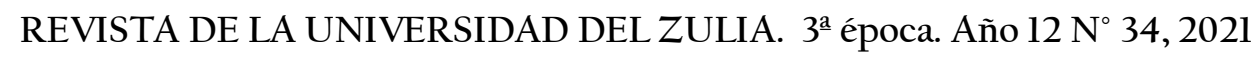

Mikel Ugando Peñate et al.// Modelización financiera aplicada en empresas... 8-28

DOI: http://dx.doi.org/10.46925//rdluz.34.02

\subsubsection{Método de la Fórmula}

El método de fórmula no es solo una manera para pronosticar los requerimientos de capital externo, sino también para llegar a conocer mediante una adecuación algebraica la tasa de crecimiento interna de las ventas, que sería soportada tanto por las fuentes propias de la empresa, o asignando un monto máximo de financiación.

El crecimiento de una entidad no solo se debe enfocar en la rapidez con la que esta puede crecer sin ningún tipo de financiamiento externo, sino en la tasa de crecimiento que se puede sostener sin emisiones adicionales de capital. Es como lo menciona en su obra Brealey, et al. (2010: 807): "si la empresa logra captar suficiente deuda, se puede financiar prácticamente cualquier tasa de crecimiento, pero tiene más sentido suponer que la empresa se sujeta a una estructura de capital óptima que conservará mientras el capital se incrementa con las utilidades retenidas". Con esto se espera que la organización solo mantenga la deuda suficiente para contener la razón deuda-capital.

\section{$F E R=\underline{A}(\Delta S)-\underline{\text { Pasivos Espontáneos }}(\Delta S)-m \times S I x(1-d)$}

\section{So SO}

\section{Donde:}

A-Activos totales

$\Delta S$ - Incremento en ventas $(S 1-S 0)=g S 0$

SO-Ventas año base

$m$-Margen de utilidad sobre ventas(EAIT/S)

$S I-$ Ventas proyectadas $(\mathrm{SO}(1+\mathrm{g}))$

$d$-razón de distribución de dividendos

Principalmente este método se usa para obtener un pronóstico aproximado y sencillo de los requerimientos financieros y como un complemento del método del balance general proyectado.

\subsection{Predicción y modelación financiera a corto plazo}

La modelación financiera se aplica en cualquier momento para que se lleve un mejor manejo de la empresa. Según lo manifiestan Ross et al., (2012: 63) existe el modelo de 


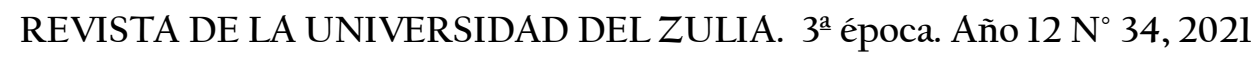

Mikel Ugando Peñate et al.// Modelización financiera aplicada en empresas... 8-28

DOI: http://dx.doi.org/10.46925//rdluz.34.02

porcentaje de ventas: "La idea básica es separar las cuentas del estado de resultados y del balance general en dos grupos, los que varían en forma directa con las ventas y los que no lo hacen".

Entonces, con esta técnica se pretende determinar un pronóstico de ventas a futuro y cuánto de financiamiento la empresa necesita para poder apoyar al nivel de ventas esperadas. Con esta forma se plantea desarrollar de manera rápida y práctica la aplicación de estados financieros pro forma, dando así un mejor esquema de predicción en el área de ventas y por ende para toda la empresa. Todo lo anterior indicado se contrasta con la investigación para un grupo de empresas del sector manufacturero expuesto por Valladares, et al., 2021.

\subsection{Predicciones y regresión lineal múltiple}

La regresión lineal se refiere a la predicción del valor de una variable a partir de una o más variables. En este modelo estadístico se pretende identificar mencionadas variables que son generalmente $\mathrm{x}, \mathrm{y}$. Donde $\mathrm{y}$ depende de $\mathrm{x}$ diferenciando así la variable dependiente (y) y la independiente $(\mathrm{x})$.

El análisis de regresión lineal múltiple como lo expresa en su obra Ugando et al. (2018) "está relacionado con el estudio de la dependencia de una variable, la variable dependiente de una o más variables adicionales". En este sentido, la regresión puede usarse como sentido de predicción en una ecuación lineal donde se ven afectadas las dos variables. Con ello se puede utilizar diagramas de dispersión y también el coeficiente de correlación r, para determinar si existe algún tipo de relación entre dos variables.

Las estimaciones de mínimos cuadrados son funciones de los datos experimentales; existen momentos en donde estos datos cambiarán de acuerdo con la muestra, es decir, pueden variar de una muestra a otra. Según lo expresa (Gujarati \& Porter, 2010) “se requiere alguna medida de confiabilidad o precisión de los estimadores $\beta 1$ y $\beta 2$. En estadística, la precisión de un valor estimado se mide por su error estándar (ee)". El error mencionado es la desviación estándar de la distribución muestral del estimador, y la distribución muestral de un estimado es tan sólo una probabilidad o distribución de frecuencias del estimador. El error se lo determina con la siguiente fórmula:

$$
e e(\beta 1)=\frac{\sqrt{\Sigma x^{2}}}{n \Sigma} \sigma(1)
$$




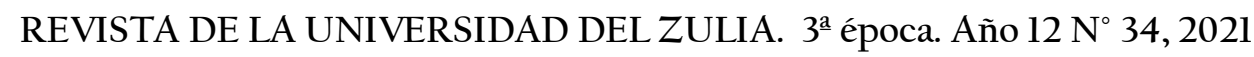

Mikel Ugando Peñate et al.// Modelización financiera aplicada en empresas... 8-28

DOI: http://dx.doi.org/10.46925//rdluz.34.02

Entonces, con esta fórmula al presentar datos estimados, se pretender encontrar la desviación estándar de los valores de Y, que se encuentra al contorno de la línea de regresión que se estima en el supuesto. Dentro del análisis de la regresión múltiple se ubican varias pruebas, como lo son Prueba Fisher y R2 de Pearson. Entre ellas se encuentra una relación precisa en el análisis de la varianza. El coeficiente de determinación R2, que se define como la proporción de la varianza total de la variable explicada por la regresión, en donde, cuanto más cerca este el resultado de 1, mayor será el ajuste del modelo a la variable de estudio. Por otra parte, la prueba de Fisher resuelve mediante el proceso de los modelos de regresión, los cuales restringe uno o más coeficientes de regresión. Estos análisis serán realizados de acuerdo a los criterios expuestos por: Rubio (2017), Sabando et al., (2020) y Ugando et al., (2019).

\section{Materiales y métodos}

En esta investigación se empleó un enfoque mixto, cuantitativo y cualitativo, siguiendo los criterios de Hernández, et al., (2014). Se utilizaron los estados financieros de la empresa y la información proporcionada por la gerencia de la misma y sus funcionarios. Teniendo en cuenta un tipo de investigación histórica y descriptiva para un periodo de tiempo de 4 años. Además, con un diseño de investigación acción que sustenta los hechos pasados para poder dar solución al problema de investigación.

La población estuvo conformada por la gerencia y el departamento de contabilidad de la empresa Reciplacom S.A, considerando todos los estados financieros para los periodos 2016-2019, así como la información económica financiera que sea susceptible de utilizar. La muestra estuvo determinada por toda la información económica financiera y los Estados Financieros, en la cual se aplicaron las técnicas de planeación y modelación financiera para la determinación de niveles de activos, las necesidades de financiamiento externo y previsiones financieras con vista a proyecciones de ventas a mediano y largo plazo.

Una de las técnicas para la recogida de datos que se vio conveniente utilizar fue la entrevista, ya que se tiene un contacto directo con las personas que se consideran fuente de información, en este caso el gerente y el departamento de contabilidad. Para poder acceder a la información necesaria se procedió a realizar grupos de preguntas para obtener información acerca de la empresa y las percepciones que tiene el departamento económico y la contadora, 


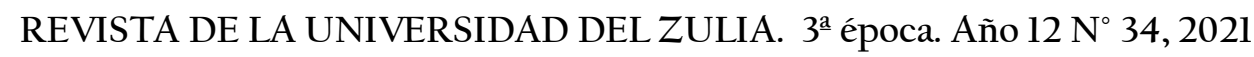

Mikel Ugando Peñate et al.// Modelización financiera aplicada en empresas... 8-28

DOI: http://dx.doi.org/10.46925//rdluz.34.02

de acuerdo a la aplicación de técnicas y herramientas de planeación y modelación financiera aplicada, todo esto corresponde a una información de tipo cualitativa.

La obtención de información de carácter cuantitativo que corresponde a los datos numéricos, como el empleo de los estados financieros de la empresa durante el periodo 2016 2019, sirvieron para aplicar las técnicas y herramientas de planeación y modelación financiera. Además, se emplearon las técnicas estadísticas y de análisis matemático a partir del uso de las aplicaciones informáticas Microsoft ${ }^{\circledR}$ Excel (2019), y SPSS versión 21.

\section{Resultados}

3.1. Diagnóstico de la situación financiera a corto plazo de la Empresa Reciplacom S.A para el período 2016-2019

Reciplacom S.A. es una empresa ecuatoriana, con sede principal en Guayaquil. Opera en tratamiento y eliminación de residuos industriales. Fue fundada el 24 de noviembre de 2014; está ubicada en Av. De los Colonos, S/N Intersección: By Pass Santo Domingo de los Tsáchilas y su actividad principal es la venta al por mayor de desperdicios, desechos y materiales de reciclamiento. Entre sus principales clientes podemos destacar: Repapers Reciclaje del Ecuador S.A. (mayor porcentaje de ventas, 65\%), Industrial Papelera Ecuatoriana S.A., Acería del Ecuador C.A., Acerías Nacionales del Ecuador S.A., Ecoresa Ecología Reciclable S.A., Intercia S.A. y Productos Familia Sancela del Ecuador S.A. El diagnóstico de la situación financiera de la empresa se presentó a través del compendio de los criterios expuestos por varios autores, tales como: Rodríguez (2012), Salvá (2014) y Pereira (2012), donde se procedió al examen de la información obtenida para el periodo 2016-2019.

3.2 Determinación de las necesidades de financiamiento externo y activos corrientes a partir de las técnicas y herramientas de planeación financiera

Utilizando los pronósticos para datos estacionarios con el fin de estimar el valor medio de datos históricos, se tomaron en cuenta las ventas del periodo 2016-2019 obteniendo un promedio de crecimiento del $10 \%$. Debido a la estacionalidad y tendencia de las ventas, la relación existente con el efectivo y equivalentes de efectivo, cuentas y documentos por cobrar, y cuentas por pagar, junto con la aplicación de la regresión lineal múltiple y ayuda del modelo 


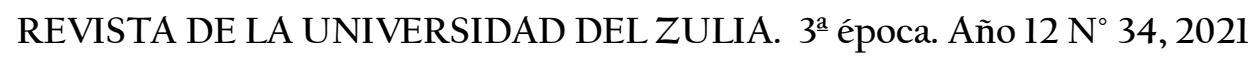

Mikel Ugando Peñate et al.// Modelización financiera aplicada en empresas... 8-28

DOI: http://dx.doi.org/10.46925//rdluz.34.02

estadístico de ARIMA, se determinó un valor de proyección del 7\% de crecimiento pesimista y un 13\% de proyección optimista (Constanza et al., 2012).

Con los resultados arrojados por la regresión lineal múltiple se pudieron establecer las proyecciones de los estados financieros para el año 2020, teniendo en cuenta 2 escenarios del $7 \%$ y 13\%, siguiendo los criterios de Ugando et al. (2019). Estos valores se tomaron en cuenta para determinar los valores de activos corrientes proyectados, teniendo en cuenta un valor de $\$ 192.831,90$ al 7\% y de $\$ 203.644,90$ al 13\% de acuerdo con los datos históricos de las ventas, cuentas por cobrar, cuentas por pagar, efectivo y equivalentes, afectando a los grupos de cuentas de la partida corriente, según se muestra en la tabla 1.

Mediante la aplicación de la ecuación (Activos - Pasivos + Patrimonio), se obtuvo como resultado para la proyección del 7\% como un escenario pesimista, que los fondos externos requeridos (FER) ascendieron a $-\$ 15,808.39$; por su parte el incremento del 13\% para un escenario optimista arrojó un valor de FER, que corresponde a \$-29,358.48, obteniéndose un exceso de financiamiento para ambos casos.

Tabla 1. Estado Proforma. Resumen de situación al cierre 2019 y proyección 2020 al 7\% y 13\%

\begin{tabular}{|c|c|c|c|}
\hline Cuentas & Año 2019 & $\begin{array}{c}\text { Proyección } \\
7 \%\end{array}$ & $\begin{array}{c}\text { Proyección } \\
13 \%\end{array}$ \\
\hline $\begin{array}{c}\text { Activos } \\
\text { corrientes }\end{array}$ & 180216.73 & $192,831.90$ & 203644.90 \\
\hline $\begin{array}{l}(+) \text { Activos no } \\
\text { corrientes }\end{array}$ & 62467.42 & 62467.42 & 62467.42 \\
\hline $\begin{array}{l}(=) \text { Total } \\
\text { activos }\end{array}$ & 242684.15 & 255299.32 & 266112.32 \\
\hline $\begin{array}{l}(-) \text { Total } \\
\text { pasivos }\end{array}$ & 172267.66 & $180,950.84$ & $188,393.57$ \\
\hline $\begin{array}{c}(-) \text { Total } \\
\text { patrimonio }\end{array}$ & 70416.49 & $90,156.87$ & $107,077.23$ \\
\hline $\begin{array}{l}\text { (-) Total de } \\
\text { Pasivo y } \\
\text { Patrimonio }\end{array}$ & 49273.93 & $271,107.71$ & $295,470.80$ \\
\hline $\begin{array}{c}\text { Total, de pasivo } \\
\text { y patrimonio }\end{array}$ & & & $590,941.60$ \\
\hline $\begin{array}{c}(=) \text { FER } \\
\text { (Exceso de } \\
\text { financiamiento) }\end{array}$ & & $-15,808.39$ & $-29,358.48$ \\
\hline
\end{tabular}

Fuente: elaboración propia a través del análisis de los Estados financieros. 
REVISTA DE LA UNIVERSIDAD DEL ZULIA. 3é época. Año $12 \mathrm{~N}^{\circ}$ 34, 2021

Mikel Ugando Peñate et al.// Modelización financiera aplicada en empresas... 8-28

DOI: http://dx.doi.org/10.46925//rdluz.34.02

Método de Fórmulas para la determinación de los Fondos Externos Requeridos

-Proyección del 7\%

$$
\begin{aligned}
& \boldsymbol{F E} \boldsymbol{R}=\frac{A(\Delta S)}{S 0}-\frac{\text { Pasivos Espontáneos }(\Delta S)}{S 0}-m x S 1 x(1-d) \\
& \boldsymbol{F E} \boldsymbol{R}=\frac{192831.990(69518.16)}{993116.51}-\frac{132728.64(69518.16)}{993116.51}-19740.38
\end{aligned}
$$

\section{$F E R=-15534.53$}

- Proyección del $13 \%$

$$
\begin{aligned}
& \boldsymbol{F} \boldsymbol{E} \boldsymbol{R}=\frac{A(\Delta S)}{S 0}-\frac{\text { Pasivos Espontáneos }(\Delta S)}{S 0}-m x S 1 x(1-d) \\
& \boldsymbol{F E} \boldsymbol{R}=\frac{203644.90(129105.15)}{993116.51}-\frac{14171.37(129105.15)}{993116.51}-36660.74
\end{aligned}
$$

\section{$F E R=-28410.92$}

3.3 Análisis de las previsiones financieras mediante la modelación financiera aplicada y el uso de la estadística descriptiva

Al realizar las pruebas de normalidad y aleatoriedade, según el estadístico Kolmogorv Smirnov, se tiene que el grado de significación es 0,000. No se puede probar la normalidad, se tendría que eliminar datos extremos que están alejados de su media. Esto nos indica que las ventas tienen una alta volatilidad, porque las ventas no siguen una distribución normal, lo cual nos permite presenciar que en ciertas épocas del año existen incrementos de las ventas (según se muestra en Tabla 2 y la figura l) de componente de factor estacional, para ingresos por ventas. De igual forma se muestra en tabla 4 el análisis de correlaciones de Pearson de las variables explicativas efectivo y equivalentes de efectivo, cuentas por cobrar, cuentas por pagar en función de las ventas, donde se identifica la mayor significación del modelo con respecto a la variable cuentas por cobrar. 
REVISTA DE LA UNIVERSIDAD DEL ZULIA. $3^{a}$ época. Año $12 \mathrm{~N}^{\circ}$ 34, 2021

Mikel Ugando Peñate et al.// Modelización financiera aplicada en empresas... 8-28

DOI: http://dx.doi.org/10.46925//rdluz.34.02

Tabla 2. Prueba de la normalidad y aleatoriedad residual de las ventas por efecto de las cuentas efectivo y equivalente de efectivo, cuentas por cobrar y cuentas por pagar

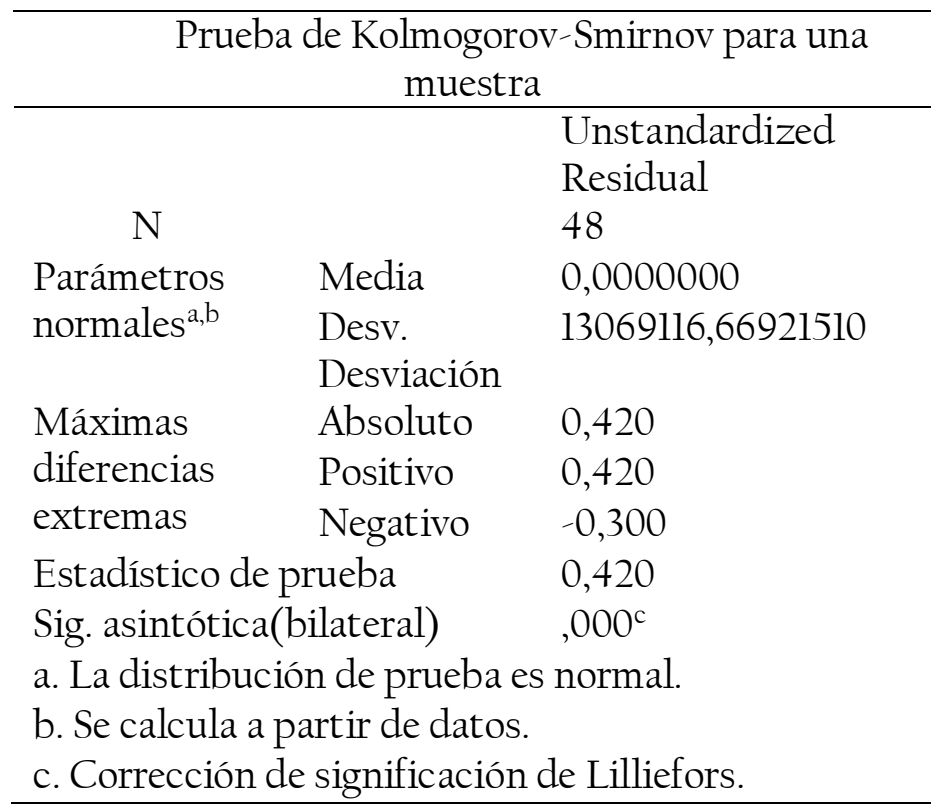

\begin{tabular}{ll}
\hline \multicolumn{2}{c}{ Prueba de rachas } \\
\hline & $\begin{array}{l}\text { Unstandardized } \\
\text { Residual }\end{array}$ \\
Valor de prueba & \multicolumn{1}{c}{828647,65678} \\
Casos < Valor de & 24 \\
prueba & \\
Casos > = Valor de & 24 \\
prueba & \\
Casos totales & 48 \\
Número de rachas & 10 \\
$Z$ & $-4,231$ \\
Sig. & 0,000 \\
asintótica(bilateral) & \\
a. Mediana & \\
\end{tabular}

Fuente: elaboración propia a través del procesamiento de información y aplicación del SPSS versión 21 .

Tabla 3. Correlación de Pearson de las variables explicativas efectivo y equivalentes de efectivo, cuentas por cobrar, cuentas por pagar en función de las ventas

\begin{tabular}{|c|c|c|c|c|c|}
\hline \multicolumn{6}{|c|}{ Análisis de Correlaciones de Pearson } \\
\hline \multirow{4}{*}{$\begin{array}{c}\text { Ingresos por } \\
\text { ventas }\end{array}$} & & $\begin{array}{c}\text { Ingresos } \\
\text { por ventas }\end{array}$ & $\begin{array}{c}\text { Efectivo y } \\
\text { equivalente de } \\
\text { efectivo }\end{array}$ & $\begin{array}{c}\text { Cuentas y } \\
\text { documentos } \\
\text { por cobrar }\end{array}$ & $\begin{array}{c}\text { Cuentas por } \\
\text { pagar }\end{array}$ \\
\hline & $\begin{array}{c}\text { Correlación de } \\
\text { Pearson }\end{array}$ & 1 & $-0,033$ &, $729 * *$ & 0,033 \\
\hline & Sig. (bilateral) & & 0,825 & 0,000 & 0,825 \\
\hline & $\mathrm{N}$ & 48 & 48 & 48 & 48 \\
\hline \multirow{3}{*}{$\begin{array}{c}\text { Efectivo y } \\
\text { equivalente de } \\
\text { efectivo }\end{array}$} & $\begin{array}{l}\text { Correlación de } \\
\text { Pearson }\end{array}$ & $-0,033$ & 1 & $-0,017$ & 0,221 \\
\hline & Sig. (bilateral) & 0,825 & & 0,910 & 0,132 \\
\hline & $\mathrm{N}$ & 48 & 48 & 48 & 48 \\
\hline \multirow{3}{*}{$\begin{array}{l}\text { Cuentas y } \\
\text { documentos } \\
\text { por cobrar }\end{array}$} & $\begin{array}{c}\text { Correlación de } \\
\text { Pearson }\end{array}$ &, $729 * *$ & $-0,017$ & 1 & $-0,046$ \\
\hline & Sig. (bilateral) & 0,000 & 0,910 & & 0,759 \\
\hline & $\mathrm{N}$ & 48 & 48 & 48 & 48 \\
\hline \multirow[t]{3}{*}{$\begin{array}{c}\text { Cuentas por } \\
\text { pagar }\end{array}$} & $\begin{array}{c}\text { Correlación de } \\
\text { Pearson }\end{array}$ & 0,033 & 0,221 & $-0,046$ & 1 \\
\hline & Sig. (bilateral) & 0,825 & 0,132 & 0,759 & \\
\hline & $\mathrm{N}$ & 48 & 48 & 48 & 48 \\
\hline
\end{tabular}

**. La correlación es significativa en el nivel 0,01 (bilateral). 
REVISTA DE LA UNIVERSIDAD DEL ZULIA. $3^{a}$ época. Año $12 \mathrm{~N}^{\circ}$ 34, 2021

Mikel Ugando Peñate et al.// Modelización financiera aplicada en empresas... 8-28

DOI: http://dx.doi.org/10.46925//rdluz.34.02

Siguiendo el análisis se procedió a determinar el índice de tolerancia y el factor de la inflación de las variables. Para el primer caso se identifica un índice de tolerancia superior a 0,1 y además el factor de la inflación de la varianza (FIV) no supera el rango de 5 a 10, por lo tanto no existe la presencia de la colinealidad y la multicolinealidad, según resultados de la tabla 5.

De igual forma se evalúo el supuesto de la independencia de los residuos propuestos por Durbin y Watson, arrojando un valor de 1.964, y este a la vez se ubica dentro del rango permitido de la independencia de los residuos de 1,5 a 2,5. De esta forma, se cumple con tal rigurosidad cada uno de los procesos de los supuestos para someter a las variables explicativas y resultados a un modelo estadístico, que se ajusta lo más real posible al índice de predicción.

Figura 1. Componente del Factor Estacional para el nivel de ventas

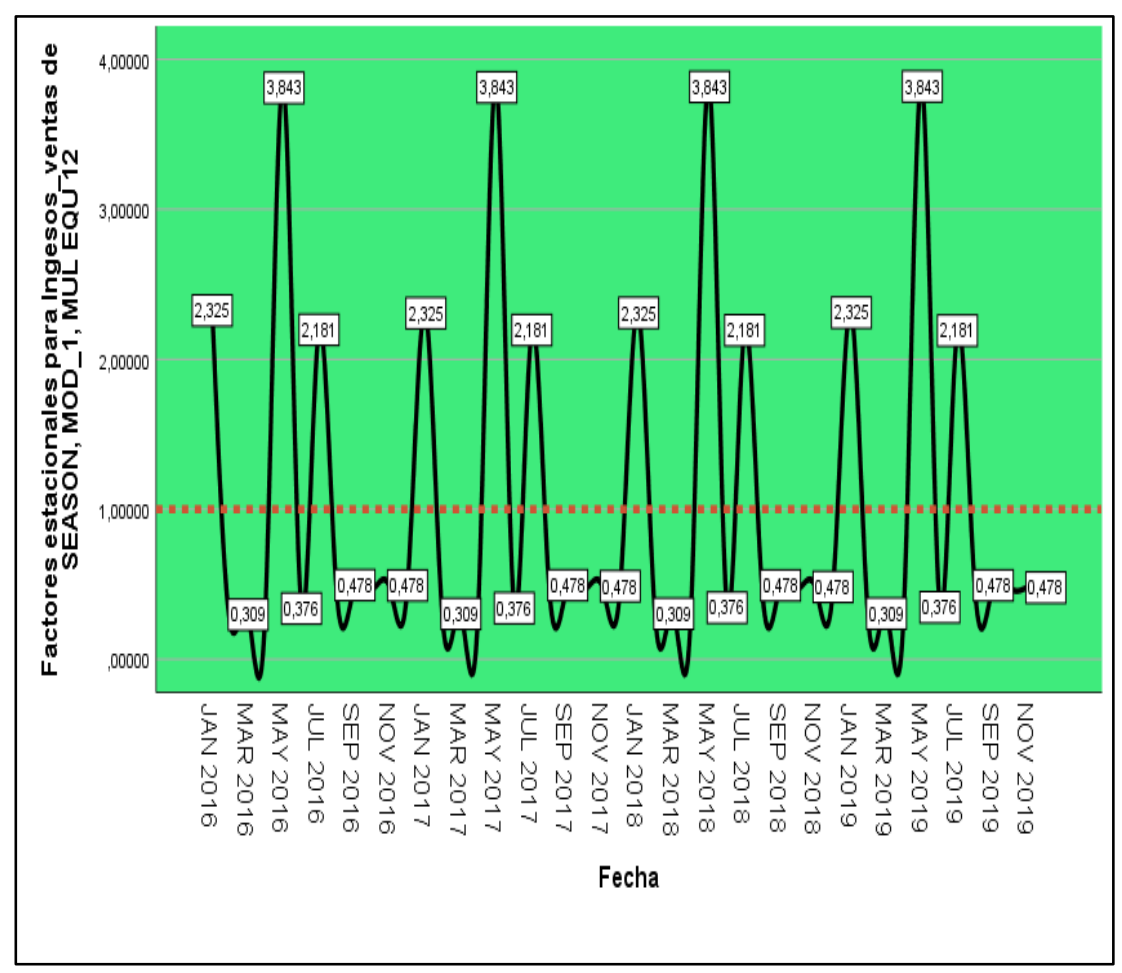

\begin{tabular}{|c|c|}
\hline \multicolumn{2}{|c|}{ Factores estacionales } \\
\hline $\begin{array}{c}\text { Nombre de } \\
\text { serie: }\end{array}$ & $\begin{array}{c}\text { Ingresos por } \\
\text { ventas }\end{array}$ \\
\hline Período & $\begin{array}{c}\text { Factor } \\
\text { estacional } \\
(\%)\end{array}$ \\
\hline 1 & 232,5 \\
\hline 2 & 24,3 \\
\hline 3 & 30,9 \\
\hline 4 & 34,6 \\
\hline 5 & 384,3 \\
\hline 6 & 37,6 \\
\hline 7 & 218,1 \\
\hline 8 & 47,0 \\
\hline 9 & 47,8 \\
\hline 10 & 47,2 \\
\hline 11 & 48,0 \\
\hline 12 & 47,8 \\
\hline
\end{tabular}

Fuente: elaboración propia a través del procesamiento de información y aplicación del SPSS versión 21 . 
REVISTA DE LA UNIVERSIDAD DEL ZULIA. 3época. Año 12 N³4, 2021

Mikel Ugando Peñate et al.// Modelización financiera aplicada en empresas... 8-28

DOI: http://dx.doi.org/10.46925//rdluz.34.02

Tabla 4. Modelación lineal múltiples del efectivo y equivalentes de efectivo, cuenta por cobrar, cuenta por pagar sobre las ventas

\begin{tabular}{|c|c|c|c|c|c|c|c|c|}
\hline \multirow[t]{2}{*}{ Modelo } & \multirow{2}{*}{$\begin{array}{c}\text { Coeficientes } \\
\text { no } \\
\text { estandarizados } \\
\text { B }\end{array}$} & \multirow{2}{*}{$\begin{array}{r}\text { Coeficientes } \\
\text { estandarizados } \\
\text { Beta }\end{array}$} & \multirow[t]{2}{*}{$t$} & \multirow[t]{2}{*}{ Sig. } & \multicolumn{2}{|c|}{$\begin{array}{c}95,0 \% \text { intervalo de } \\
\text { confianza } \\
\text { para B }\end{array}$} & \multicolumn{2}{|c|}{$\begin{array}{c}\text { Estadísticas de } \\
\text { colinealidad }\end{array}$} \\
\hline & & & & & L. Inferior & L. Superior & Tolerancia & VIF \\
\hline (Constante) & $-288707,6$ & & 0,082 & 0,935 & $-7354832,16$ & 6777416,84 & & \\
\hline $\begin{array}{l}\text { Efectivo y } \\
\text { equivalente de } \\
\text { efectivo }\end{array}$ & $-3,430$ & $-0,037$ & 0,350 & 0,728 & $-23,177$ & 16,316 & 0,951 & 0,051 \\
\hline $\begin{array}{l}\text { Cuentas y } \\
\text { documentos } \\
\text { por cobrar }\end{array}$ & 8,567 & 0,732 & 0,127 & 0,000 & 6,144 & 10,989 & 0,998 & 0,002 \\
\hline $\begin{array}{l}\text { Cuentas por } \\
\text { pagar }\end{array}$ & 13,914 & 0,074 &, 705 & 0,485 & 25,884 & 53,712 & 0,950 & 0,053 \\
\hline
\end{tabular}

Fuente: elaboración propia a través del procesamiento de información y aplicación del SPSS versión 21.

El modelo de regresión lineal múltiple obtenido fue el siguiente: Y= -288707,660 $3,430 \mathrm{X} 1+8,567 \mathrm{X} 2+13,914 \mathrm{X} 3$, acompañado de una covarianza $73,30 \%$ y un coeficiente de determinación ajustado $\mathrm{R}^{2}=53,70 \%$, tomando en consideración el análisis de las varianzas (ANOVA) según el Método de Fisher de F=17,01 y un nivel de significancia ( $p<0,001)$. De la misma manera, el coeficiente tipificado Beta reportó para cada variable independente su fuerza sobre las ventas $(7,40 \%, 73,20 \%$ y $-3,70 \%)$, siendo las cuentas por cobrar la variable que más se destaca. Por consiguiente, lo justifica la Prueba bondad de ajuste $\mathrm{T}$ de student ( $\mathrm{T}=$ $7,127 ; \mathrm{p}=0,000)$ con un valor menor al $5 \%$ de significancia $(\mathrm{p}<0,05)$, respectivamente para este estudio. Demostrando estas pruebas de bondades de ajuste y además que los coeficientes no estandarizados son diferentes de cero (0), la confiabilidad de este modelo estadístico para llevar a cabo la predicción financeira (ver tabla 5 del resumen del modelo).

\subsection{Análisis de las predicciones a través del empleo del Modelo ARIMA}

En referente al comportamiento de la predicción de los Ingresos por Ventas, Efectivo y Equivalentes de Efectivo, Cuentas y Documentos por Cobrar y Cuentas por Pagar en miles de dólares, consideradas como las variables endógenas (según se muestra en las tablas 6, 7 y 8), se obtuvo un modelo autoregresivo integrado de media móvil ARIMA $(0,0,0)(0,0,0)$, para los Ingresos con sus respectivos parámetros que intervienen durante el proceso de la predicción 
REVISTA DE LA UNIVERSIDAD DEL ZULIA. 3e época. Año $12 \mathrm{~N}^{\circ}$ 34, 2021

Mikel Ugando Peñate et al.// Modelización financiera aplicada en empresas... 8-28

DOI: http://dx.doi.org/10.46925//rdluz.34.02

como: MAE (5.502.789,167), MaxAPE (1.350,499), MaxAE $(104.106 .303,7)$ y el índice de coeficiente Bayesiano (BIC=33,652), y a la vez autorregresivo de acuerdo la prueba de LjungBox Q(18) presentó un valor de significancia de 0,946 (P>0,05), demostrando para la predicciones de los Ingresos la presencia de ruido blanco. Con respecto a la modelación del Efectivo y Equivalentes de Efectivo se evidencia un $\operatorname{ARIMA}(0,0,0)(0,0,0)$ con sus respectivos parámetros que intervienen durante este proceso de la predicción como: MAE $(71.452,829)$, MaxAPE (2.578,635), MaxAE (1.020.625,360) y el índice de coeficiente Bayesiano (BIC=24,575) y la prueba de Ljung-Box Q (18) presentó un valor de significancia de 0,982 $(\mathrm{P}>0,05)$, dado este caso manifiesta la presencia de ruido blanco. La modelación de las Cuentas y Documentos por cobrar en miles de dólares se evidencia un $\operatorname{ARIMA}(0,0,0)(0,0,0)$ con sus respectivos parámetros que intervienen durante este proceso de la predicción como: MAE (427.269,111), MaxAPE (1.216,621), MaxAE $(10.834 .140,52)$ y el índice de coeficiente Bayesiano (BIC=28,721) y la prueba de Ljung-Box Q (18) presentó un valor de significancia de 0,853 (P>0,05), dado este caso manifiesta la presencia de ruido blanco.

Tabla 5. Modelación lineal múltiple del Efectivo y equivalentes de efectivo, Cuentas por cobrar, cuentas por pagar sobre las ventas (ANOVA).

\section{ANOVA $^{\mathrm{a}}$}

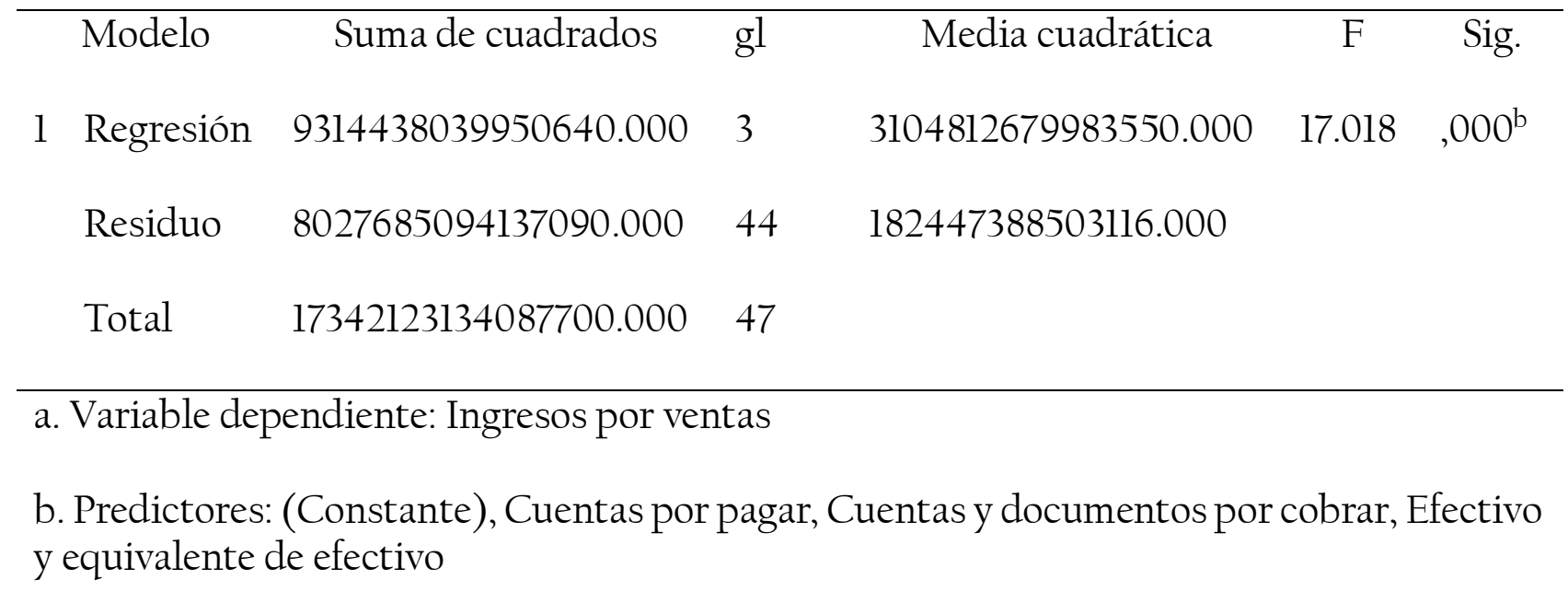


REVISTA DE LA UNIVERSIDAD DEL ZULIA. 3 época. Año $12 \mathrm{~N}^{\circ}$ 34, 2021

Mikel Ugando Peñate et al.// Modelización financiera aplicada en empresas... 8-28

DOI: http://dx.doi.org/10.46925//rdluz.34.02

Tabla 6. Fluctuación del modelo ARIMA

\section{Descripción del modelo}

\begin{tabular}{llll}
\hline ID de modelo & & Ingresos por ventas & Mipo de modelo \\
Efectivo y equivalente de & Modelo_2 & ARIMA $(0,0,0)(0,0,0)$ \\
efectivo & ARIMA $(0,0,0)(0,0,0)$ \\
$\begin{array}{l}\text { Cuentas y documentos } \\
\text { por cobrar } \\
\text { Cuentas por pagar }\end{array}$ & Modelo_3 & ARIMA $(0,0,0)(0,0,0)$ \\
& Modelo_4 & ARIMA $(2,1,0)(0,0,0)$ \\
\hline
\end{tabular}

Fuente: elaboración propia a través del procesamiento de información y aplicación del SPSS versión 21.

Por último, en la modelación de las Cuentas por Pagar se obtuvo un ARIMA $(2,1,0)$ $(0,0,0)$. Este resultado nos permite inferir a lo largo del tiempo dos patrones de autocorrelación y de igual manera una presencia integrada de la independencia y la estacionalidad. En este sentido, los parámetros que intervienen durante este proceso de la predicción, como: MAE $(9.267,984)$, MaxAPE $(38,013)$, MaxAE $(42.785,250)$ y el índice de coeficiente Bayesiano (BIC=19,263) y la prueba de Ljung-Box Q (17), presentó un valor de significancia de 0,975 (P>0,05), dado este caso manifiesta la presencia de ruido blanco. Vale recalcar que para los Estados Financieros de la empresa, a través del tiempo se manifiesta un escenario alentador en cada uno de sus procesos econométricos.

En cuanto al análisis de la predicción empleando el Modelo ARIMA, se evidencia que la variable Ingresos por Ventas manifiesta un valor constante desde enero del año 2020 hasta el cierre del año 2021 de \$ 1, 955,614.28. Con respecto a la variable Efectivo y Equivalentes de Efectivo, se puede apreciar un valor constante de inicio del 2020 hasta el cierre del año 2021 de $\$ 32,342.64$. De igual manera se comprueba que las variaciones de las demás variables de estudio son constantes a través del tiempo, creando confianza para los pronósticos, siendo estos resultados como apoyo para trazar estrategias respecto a la planeación, proyecciones y el desempeño de los funcionarios en la obtención de mejores resultados futuros respecto a la inversión. 
REVISTA DE LA UNIVERSIDAD DEL ZULIA. 3é época. Año $12 \mathrm{~N}^{\circ}$ 34, 2021

Mikel Ugando Peñate et al.// Modelización financiera aplicada en empresas... 8-28

DOI: http://dx.doi.org/10.46925//rdluz.34.02

Tabla 7. Comportamiento del modelo ARIMA de los Ingresos por Ventas, Efectivo y Equivalentes de Efectivo, Cuentas y Documentos por Cobrar y Cuentas por Pagar en miles de dólares en función de ajuste del modelo desde el año 2016 hasta el 2021.

\begin{tabular}{|c|c|c|c|c|c|c|c|}
\hline Modelo & $\begin{array}{c}\mathrm{R} \\
\text { cuadrado } \\
\text { estacionaria }\end{array}$ & $\begin{array}{c}\mathrm{R} \\
\text { cuadrado }\end{array}$ & RMSE & MAPE & MAE & $\begin{array}{l}\text { Max } \\
\text { APE }\end{array}$ & $\begin{array}{c}\text { Max } \\
\mathrm{AE}\end{array}$ \\
\hline $\begin{array}{l}\text { Ingresos por } \\
\text { ventas- } \\
\text { Modelo }\end{array}$ & $3.930 \mathrm{E}-14$ & -0.030 & 19492927.6 & 241.04 & 5502789.16 & 1350.49 & 104106303.7 \\
\hline $\begin{array}{l}\text { Efectivo y } \\
\text { equivalente } \\
\text { de efectivo- } \\
\text { Modelo_2 }\end{array}$ & $1.554 \mathrm{E}-14$ & -0.022 & 208418.33 & 521.32 & 71452.829 & 2578.63 & 1020625.36 \\
\hline $\begin{array}{l}\text { Cuentas y } \\
\text { documentos } \\
\text { por cobrar- } \\
\text { Modelo_3 }\end{array}$ & $7.361 \mathrm{E}-14$ & -0.019 & 1656129.09 & 202.38 & 427269.111 & 1216.62 & 10834140.52 \\
\hline $\begin{array}{l}\text { Cuentas por } \\
\text { pagar- } \\
\text { Modelo_4 }\end{array}$ & 0.192 & 0.979 & 14628.647 & 7.296 & 9267.984 & 38.013 & 42785.25 \\
\hline
\end{tabular}

Fuente: elaboración propia a través del procesamiento de información y aplicación del SPSS versión 21.

Tabla 8. Autocorrelación del estadístico Q (Ljung-Box) desde el año 2016 hasta el 2021.

\begin{tabular}{cccc}
$\begin{array}{c}\text { BIC } \\
\text { normalizado }\end{array}$ & Estadísticos & DF & Sig. \\
\hline 33.65 & 9.550 & 18 & 0.946 \\
24.57 & 7.726 & 18 & 0.982 \\
28.72 & 11.87 & 18 & 0.853 \\
19.26 & 7.543 & 17 & 0.975 \\
\hline
\end{tabular}

Fuente: elaboración propia a través del procesamiento de información y aplicación del SPSS versión 21.

\section{Discusión y Conclusiones}

La regresión lineal múltiple se estableció de acuerdo a las diferentes herramientas y modelos estadísticos, siguiendo los criterios de Rubio (2017) y Sabando et al. (2020); tomando en consideración que las variables explicativas no tienen una mayor incidencia a diferencia de las variables sobre las ventas. Al aplicar las pruebas de normalidad y aleatoriedad arrojan resultados no favorables ya que la distribución no es normal, en base a las variables de estudio, al realizar un enfoque más delicado de la multicolinealidad sellega a deducciones 


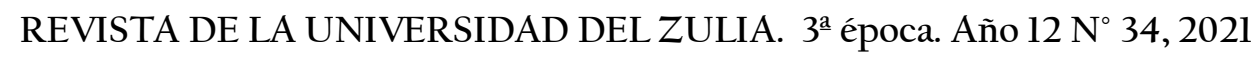

Mikel Ugando Peñate et al.// Modelización financiera aplicada en empresas... 8-28

DOI: http://dx.doi.org/10.46925//rdluz.34.02

agradables, ya que se encuentran dentro de los niveles permitidos de la independencia de los residuos. Se determinó que la mejor variable de estudio que explica el modelo son las Cuentas por Cobrar con un $(73,20 \%)$.

Al aplicar el modelo ARIMA se encontró que las ventas presentan una alta volatilidad y tendencia, siendo similar al estudio realizado por Ugando et al., (2019); donde las ventas fluctúan manteniendo relación entre las variables estudiadas. Se consideran que las ventas tendrán un comportamiento creciente a futuro, ya que al proyectar los niveles de activos en las variables estudiadas, estas crecerán por lo que se evidencia que guardan coherencia entre sí.

Se procedió a determinar las necesidades de financiamiento requeridas para la empresa; el mismo que para ser encontrado se estimaron porcentajes en escenario pesimista del $7 \%$ y un escenario optimista del 13\%, basados en la técnica de pronósticos estacionarios expuestas por Constanza et al., (2012) que incluye los datos históricos que se pueden evidenciar en el comportamiento de los estados financieros.

$\mathrm{Al}$ realizar las proyecciones pesimista y optimista se logró determinar que los niveles de activos corrientes son de $\$ 192,831.90$ y un valor de $\$ 203,644.90$, para los respectivos pronósticos. Se obtuvieron excesos de fondos externos requeridos para un nivel del $7 \%$ con un valor de $\$ 15,808.39$; y para el $13 \%$ un valor de $\$ 29,358.48$.

\section{Referencias}

Brealey, R., Myers, S., \& Allen, F. (2010). Principios de Finanzas Corporativas (Novena ed.). México: Mc Graw Hill.

Castro, Z. (2017). Evaluación de proyectos de inversión para pequeñas y medianas empresas con una estrategia de proyección financiera. Ra Ximhai, 27.

Constanza, , M., Parra, R., \& López, L. (2012). Presupuestos enfoque para la planeación financiera (Primera ed.). Colombia: PEARSON.

Córdoba, M. (2012). Gestión Financiera. Bogotá: Ecoe Ediciones.

Domínguez, M. (2011). Dirección Financiera. España: Vértice.

Fernández, E. (2010). Administración de Empresas: Un Enfoque Interdisciplinar (Primera ed.). Madrid. 
REVISTA DE LA UNIVERSIDAD DEL ZULIA. 3é época. Año $12 \mathrm{~N}^{\circ}$ 34, 2021

Mikel Ugando Peñate et al.// Modelización financiera aplicada en empresas... 8-28

DOI: http://dx.doi.org/10.46925//rdluz.34.02

Gómez, F. (2003). Dirección Financiera (Tercera ed.). Desclée de Brouwer.

Gujarati, D. N., \& C., P. D. (2010). Econometría (Quinta ed.). México, México: Mc Graw Hill.

Hernández, R., Fernández, C., \&\& Baptista, P. (2014). Metodología de la Investigación. México DF: McGraw - HILL Education.

Pereira, F. B. (2012). Contabilidad para dirección. Navarra. EUNSA.

Rodríguez, L. (2012). Análisis de Estados Financieros. México D.F: Mc Graw.

Ross, S., Westerfield, R., \& Jaffe, J. (2012). Finanzas Corporativas (Novena ed.). México: McGraw Hill.

Ross, S., Westerfield, R., \& Jordan, B. (2014). Fundamentos de Finanzas Corporativas (Décima ed.). México D.F: McGraw Hill. Interamericana Editores, S.A de C.V.

Rubio, G. (2017). Perspectiva multivariante de los pronósticos en las pymes industriales de ibagué Universidad Sergio Arboleda. Escuela de Postgrados. Maestría en producción y operaciones. Revista Facultad Ciencias Economicas V. 25 (2), 25-40,. DOI: https://doi.org/10.18359/rfce.3067

Sabando, Á, Ugando, M., Cueva, E., Villalón, A., Mendoza, G. \& Arias, J. (2020). Modelación productiva y pronósticos de las ventas del cultivo de la pitahaya en Ecuador. Sinapsis 12 (1), $94-109$.

Salvá Socías, A. R. (2014). Contabilidad Financiera. Madrid: Pirámide.

Suárez, A. (2014). Tres de Octubre. España: España Funds people. Obtenido de La planificación financiera en España y en el mundo: aún nos queda mucho que avanzar.: https://es.fundspeople.com/news/la-planificacion-financiera-en-espana-y-en-el- mundoaun-nos-queda-mucho-que-avanzar

Ugando, P., Arteaga, U., \&e Yanayn, G. (2015). Teoría del Valor Extremo (EVT), Cópulas y Valoración de riesgos. España: Académica Española de OmniScriptum GmbH \& Co. KG.

Ugando, P., Parada, G., Sixto, P., \& Yanayn, G. (2018). Planeación y Modelación Financiera del Efectivo Aplicada en Pymes de Ecuador. En Memorias CCIUTM, (Segunda Convención Científica Internacional de la Universidad Técnica de Manabí. Ecuador.

Ugando, M., Sabando, A., Zapata, A., Villalón, A., \& Sabando, B. (2019). Modelación financiera y pronósticos de fondos externos requeridos en el contexto de las pymes de Santo Domingo De Los Tsáchilas, caso Ecuador. Sinapsis, 11(2), 65-78. Obtenido de https://dialnet.unirioja.es/servlet/articulo?codigo=7399799

Ugando, P., Yanayn, G., Sabando, G., \& Villalón, P. (2019). Planeación financiera aplicada y su impacto en los resultados económicos de Pymes de Ecuador. En Memorias del II Congreso Científico Internacional FADE 2018. Ecuador: Escuela Superior Politécnica de Chimborazo. 
REVISTA DE LA UNIVERSIDAD DEL ZULIA. $3^{a}$ época. Año $12 \mathrm{~N}^{\circ}$ 34, 2021

Mikel Ugando Peñate et al.// Modelización financiera aplicada en empresas... 8-28

DOI: http://dx.doi.org/10.46925//rdluz.34.02

Ugando, M., Jurado, Z., \& Cedeño, A. (2021). Implementación de la planificación estratégica para empresas del sector automotriz en la ciudad de Santo Domingo de los Tsáchilas, ecuador: Caso importadora de Autorrepuestos Mendoza. South Florida Journal Development. Miami, v. 2, n.4, jul./sep. DOI: https://doi.org/10.46932/sfjdv2n4-006

Valladares, C., Sánchez, K., Ugando, M., Sabando, A., \& Villalón, A. (2021). Gestión de capital de trabajo y su efecto en la rentabilidad para el grupo de empresas del sector manufacturero ecuatoriano. South Florida Journal Development. Miami, v. 2, n.2, apr.jun.p. DOI: https://doi.org/10.46932/sfjdv2n2-075

Van Horne, J. W. (2010). Fundamentos de Administración Financiera. México: Pearson Educación. Van Horne, J. (2014). Fundamentos de Administración Financiera. México: Pearson Educación. 